\title{
Dual parameter measurement system for temperature and stress based on Sagnac interferomter
}

\author{
Huanhuan Yan(D, Li Wang*, Songtao Li and Jin Wang
}

Keywords: Fiber loop mirror, PCF, FBG, Temperature sensor, Stress sensing

The sensor is designed by combination of photonic crystal fiber (PCF) and fiber Bragg grating (FBG), which is based on Sagnac interferometer to measure temperature and stress simultaneously in changing environment. The interference between two parameters is avoided by cascading two wavelength moving reverse components in this sensor system. When the PCF is connected with FBG or not, the linearity of stress sensing is 0.98 and 1 , respectively. The stress sensitivity for $-0.150 \mathrm{~nm} / \mathrm{N}$ and the temperature sensitivity for $0.0113 \mathrm{~nm} /{ }^{\circ} \mathrm{C}$ are achieved on the dualparameter system. In addition, the measurement of dualparameters of temperature and stress of the complex environment is successfully realized in experiment.

\section{Introduction}

Optical fiber sensor is a device, which is used for measuring various physical parameters by the changes of physical characteristics such as interference, diffraction, polarization, reflection and loss of light caused by light propagation in the optical fiber [1-8]. The optical fiber offer many excellent characteristics such as low loss, wide frequency band, large amount of information, small size, light weight, and good winding property. However, the traditional sensor can not be used in the harsh environment of high temperature and electromagnetic field. The optical fiber sensors with the diversity structure have rapidly developed and used in different disciplines application.
Nowadays, the optical fiber sensor is widely applied in the optical communication, computer, automatic control, biomedicine, measurement, transportation, national defense and civil appliances.

There are several existing methods to measure physical parameters. The main types of optical sensors include Mach-Zehnder interferometer sensors [9, 10], surface plasmon resonance (SPR) sensors [11, 12], Fabry-Perot (FB)interferometer sensors [13], whispering gallery mode (WGM) resonator sensors [14, 15] and Sagnac interferometer sensors [16, 17]. Presently, Sagnac interferometer sensors have evolved into a powerful contender of sensing applications for multiple physical parameters because of their high reliability and sensitivity, small volume, rapid response and easy integration [18]. The sensing principle of the Sagnac interferometer sensor can be traced back to 1982 and was first studied on a nonreciprocal configuration of fiber optic gyroscope in theory and experiment by J Pavlath and Shaw [19]. The FBG can be used for stress, refractive index (RI), hydrogen and temperature sensor. Actually, The FBG sensor is more suitable for the distributed measurement due to its wavelength multipliable capability, and can be used for temperature compensation [20-22]. For PCF, the photonic band-gap fiber with air core was successfully fabricated in 1999. With the development of technology, PCF has been rapidly applied and used for the strain [23, 24], temperature [25], curvature and refractive index sensing $[26,27]$. However, the single device multiple parameters sensor is limited by the two limitation factor of

* Correspondence: Iwang.1@bjut.edu.cn

College of Applied Sciences, Beijing University of Technology, No.100

Pingleyuan, Chaoyang District, Beijing 100124, China 
cross-sensitivity and demodulation complex, which greatly hinders the development of multi-parameter sensors.

In this paper, a new experimental device is proposed which can measure the dual parameters of temperature and stress simultaneously. The PCF and FBG were composed in the hybrid structure to be used for stress and temperature sensing respectively. The corresponding characteristic peaks move to opposite direction when the stress and temperature are changing with the environment. Therefore, the mutual interference of parameters is avoided in the sensing system successfully. The designed concept has a guiding significance in the field of multi-parameter sensing.

\section{Experimental methods}

FBG is a kind of passive device, which is made by the periodic modulation of the refractive index of the fiber core. The period is usually between tens of nanometers and hundreds of nanometers. When a broad band pulse is propagated in the fiber, a narrow-band pulse is reflected while the rest is transmitted. This narrow-band pulse is called the Bragg reflection with a center wavelength $\lambda_{B}$.The Bragg reflection wavelength depends on the spacing of the refractive index modulations in the fiber. The center reflection wavelength of the FBG based on the coupling mode theory can be written as follows.

$$
\lambda_{B}=2 \mathrm{n}_{e f f} \Lambda
$$

Where $n_{\text {eff }}$ is the effective refractive index; $\Lambda$ is the grating period; $\lambda_{B}$ is Bragg wavelength. Therefore, any wavelengths that satisfied Eq. (1) will be regarded as reflection wavelengths of the FBG.

In addition, when $n_{\text {eff }}$ changes, the reflected wavelength also shifts accordingly and reflected wavelength of the FBG changes with variation of the effective refractive index. The relation of wavelength $\Delta \lambda_{B}$ and temperature $\Delta T$ is followed as,

$$
\frac{\Delta \lambda_{B}}{\lambda_{B}}=(\alpha+\xi) \Delta \mathrm{T}
$$

Where, $\Delta T$ is the thermal expansion coefficient of the fiber, $\xi=\frac{1}{n} \frac{d n}{d T}$ is the thermal optical coefficient of the fiber.

The PCF fiber loop mirror is composed of a $3 \mathrm{~dB}$ coupler, the PCF and FBG. The initial signal light enters into $3 \mathrm{~dB}$ coupler and divides into two beams: one propagating along in clockwise direction, called the forward wave; and the other one propagating counterclockwise, called the reverse wave. The two beams go through the loop propagation and then return to coupler and finally coherent output to achieve the signal light filter. The experiments are carried out based on Eqs. (1) and (2).

\section{Results and discussion}

\section{Temperature sensing}

The broadband source (BBS), $3 \mathrm{~dB}$ coupler, PCF, FBG, optical spectrum analyzer (OSA) and temperature control box (TCB) are fabricated, as shown in Fig. 1. The fiber grating and the temperature-insensitive PCF are connected to measured the temperature of the environment, which effectively avoids the cross-sensitive in multi-parameter problem.

The spectrum relation of the coupling peak intensity and the change temperature were shown in Fig. 2. We insert the enlarged image into Fig. 2 because it is not easy to distinguish the optical spectrum when the temperature is at $30,60,90,120^{\circ} \mathrm{C}$.

In Fig. 2, the broad peaks center of $1531.00 \mathrm{~nm}$ is corresponded to the characteristic peak of PCF. The narrow peaks with a range of $1550.01 \sim 1551.45 \mathrm{~nm}$ is the characteristic peak of FBG. With the increasing of temperature, the characteristic peak of FBG is red shifted obviously, while the peak of PCF does not drift. As is shown in Fig. 1, both of the PCF and FBG are in the TCB, while the red-shifted of the characteristic peak only occurred in FBG with the temperature increasing, which proves that PCF is insensitive to temperature. The

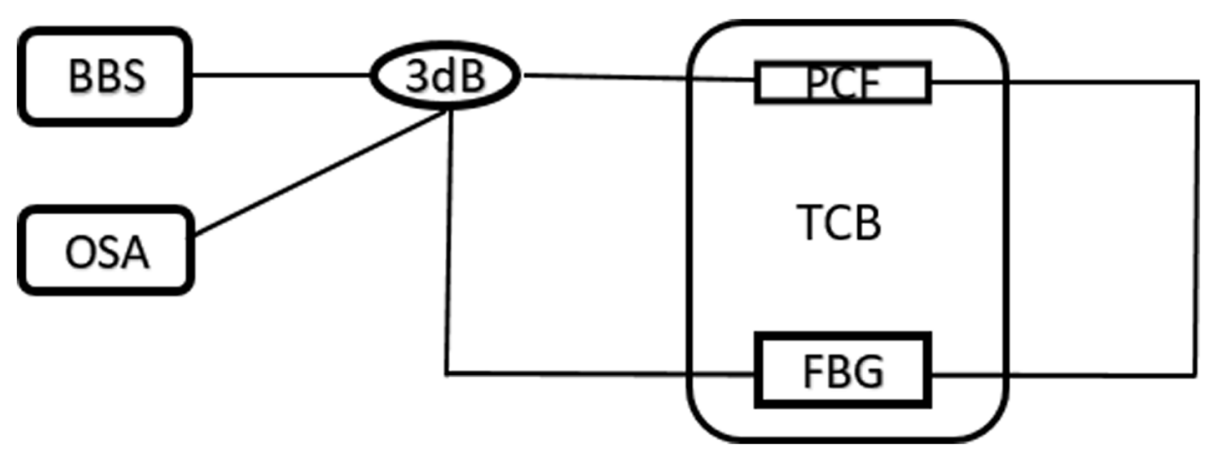

Fig. 1 Schematic of experimental principle 


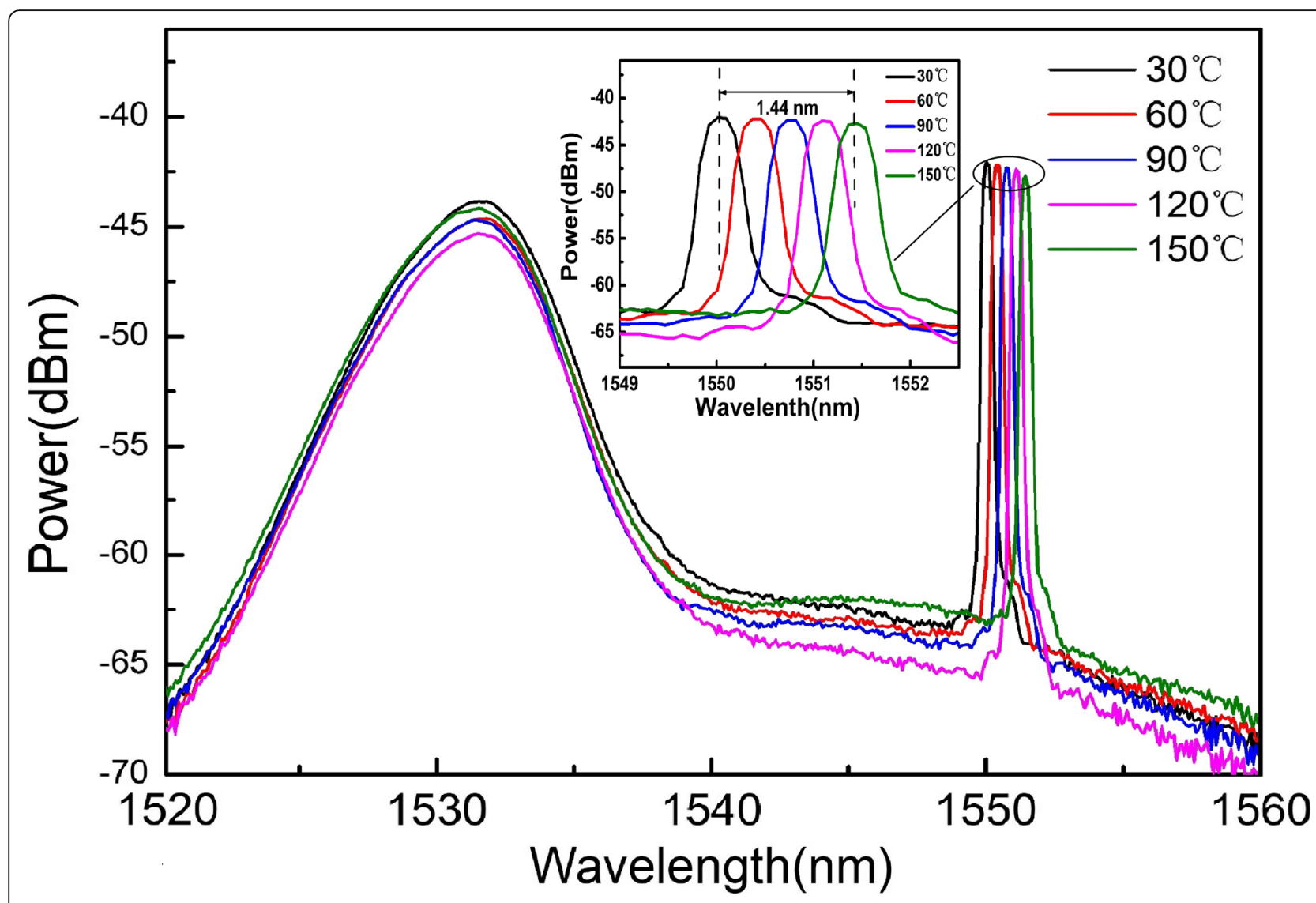

Fig. 2 The spectral curve under different temperature

waveform of the FBG does not change except redshifted at different temperatures from the inset of Fig. 2, that is to say, the temperature measurement is stable.

The central wavelengths of characteristic peaks of FBG with PCF (red dots) or not (black squares) in different temperatures are shown in Fig. 3. The black line and the red line depict the trends of wavelength changing. The peak wavelengths changes with temperature in the single FBG that is given as

$$
\begin{aligned}
& Y=0.01064 X+1549.68265 \\
& R^{2}=0.99895
\end{aligned}
$$

The peak wavelengths changes with temperature in connector of FBG and PCF that is given as

$$
\begin{aligned}
& Y=0.01127 X+1549.74193 \\
& R^{2}=0.99895
\end{aligned}
$$

The slope of the straight line of FBG and connecting FBG and PCF is 0.01064 and 0.01127 , respectively. We clearly see that the temperature sensitivity of FBG is improved by connecting with PCF. The linearity of the two straight lines are both 0.99895 . The experimental results exhibit a good repetition and stability.

To improve the performance of the dual-parameter measurement system, the FBG was coated with a thin layer of ITO by the PLD (pulsed laser deposition). The transmission spectrum and fitting line of the system after FBG coated is shown in Fig. 4. The partial enlargement of the FBG interference spectrum at different temperatures is displayed in inset (a). The fitted line graph indicated in inset (b) shows the relationship between the peak wavelength and temperature of the coated FBG.

The waveform is stable with no distortions and miscellaneous peaks, which is presented in Fig. 4. The characteristic peak of $\mathrm{FBG}$ presents red shift with the temperature increasing from 30 to $150{ }^{\circ} \mathrm{C}$ as shown in inset (a). The peak waves of the coated FBG are changing from $1549.90 \mathrm{~nm}$ to $1551.10 \mathrm{~nm}$. The results of the experiment and simulating line are highly consistent, which is depicted in inset (b). The calculated sensitivity and linearity of temperature are $0.01 \mathrm{~nm} /{ }^{\circ} \mathrm{C}$ and 1 , respectively. Therefore, the stability and the linearity of the proposed temperature measurement sensor are fully demonstrated. 


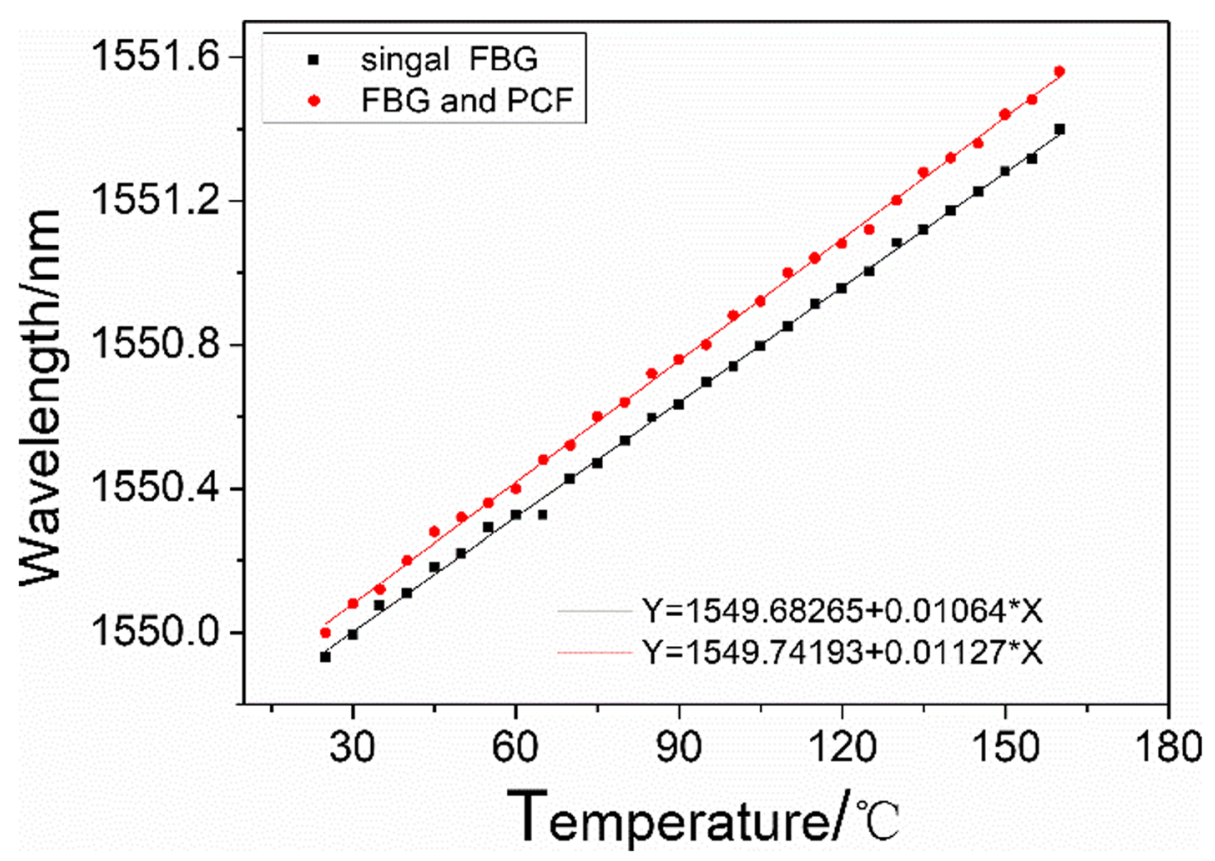

Fig. 3 Peak wavelengths versus temperature for single FBG and connector of FBG and PCF

Since ITO is a conductive and transparent semiconductor metal oxide which is sensitive to current and light, the ITO film can be used to measure refractive index, humidity and $\mathrm{pH}$. Although the temperature sensitivity was not improved, there was no significant decrease, which is indicated that coating ITO on the FBG does not have a significant effect on the measurement of its temperature. It is the method of coating ITO on FBG that provides a scientific research idea for multiparameter measurement.

\section{Pressure sensing}

Dual-parameter sensor of temperature and pressure based on Sagnac interferometer have been designed in this experiment. The principle of the pressure sensing and experimental results are analyzed as following.

In pressure sensing, the sensitive fiber is used of PCF as its peak wavelength of characteristic peak is 1531.8 $\mathrm{nm}$. The stress sensor of single PCF device which is based on Sagnac interferometer was fabricated and measured. BBS, $3 \mathrm{~dB}$ coupler, PCF, OSA and EAB are used in this experiment which is shown in Fig. 5(a). In the stress sensing experiment, we affixed the PCF to the isometric beam axis. The transmission peak is obtained in our experiment as large as possible by adjust the angle of the arms of the loop mirror and then fix it. The strength beam in this experiment is made of plexiglass. In Fig. 5(a), the beam length $\mathrm{L}$ is $306.9 \mathrm{~mm}$, the beam bottom width is $74.5 \mathrm{~mm}$, the upper bottom width $\mathrm{b}$ is $30.0 \mathrm{~mm}$ and the thickness $\mathrm{h}$ is $5.4 \mathrm{~mm}$. The stress of the PCF was changed by adding weight at the end of the beam and the change of spectrum was detected by OSA in the experiment. Figure 5(b) is the sensitive probe of PCF and Fig. 5 is the schematic diagram of the pressure experiment. The characteristic peak of PCF are shown in Fig. 6. The spectra overlaid at the weight of $0,1,2,3$, and $4 \mathrm{~N}$ are shown in Fig. 6 . Each waveform of the spectra is not affected by adding weights and the peak power corresponding to the spectra is the vicinity of $-42 \mathrm{dBm}$. The blue-shift of peak wavelength is small because of the wide spectrum. In order to design a sensitive optical fiber sensor of dual-parameter, FBG is added to the pressure sensor and the schematic diagram is shown in Fig. 7. It is used for temperature sensing by being fixed in a metal device which can protect it from stress while the PCF is applied for stress sensing in this experiment.

At room temperature, the vertical suspension weight is added to one side of the PCF arm beam, and the beam will change with the increase of the hanging weight. The PCF will bend along with the cantilever beam because it is at the center of the equal arm beam and close contact with it.

The spectral overlay curves with the gravity of $0,1,2$, 3 , and $4 \mathrm{~N}$ are shown in Fig. 8.The five peaks of the FBG are perfectly coincident in Fig. 8. As the external pressure increasing, the characteristic peaks of the PCF move to shortwave direction, which is called the blue shift. The characteristic peak of PCF, which is used for 

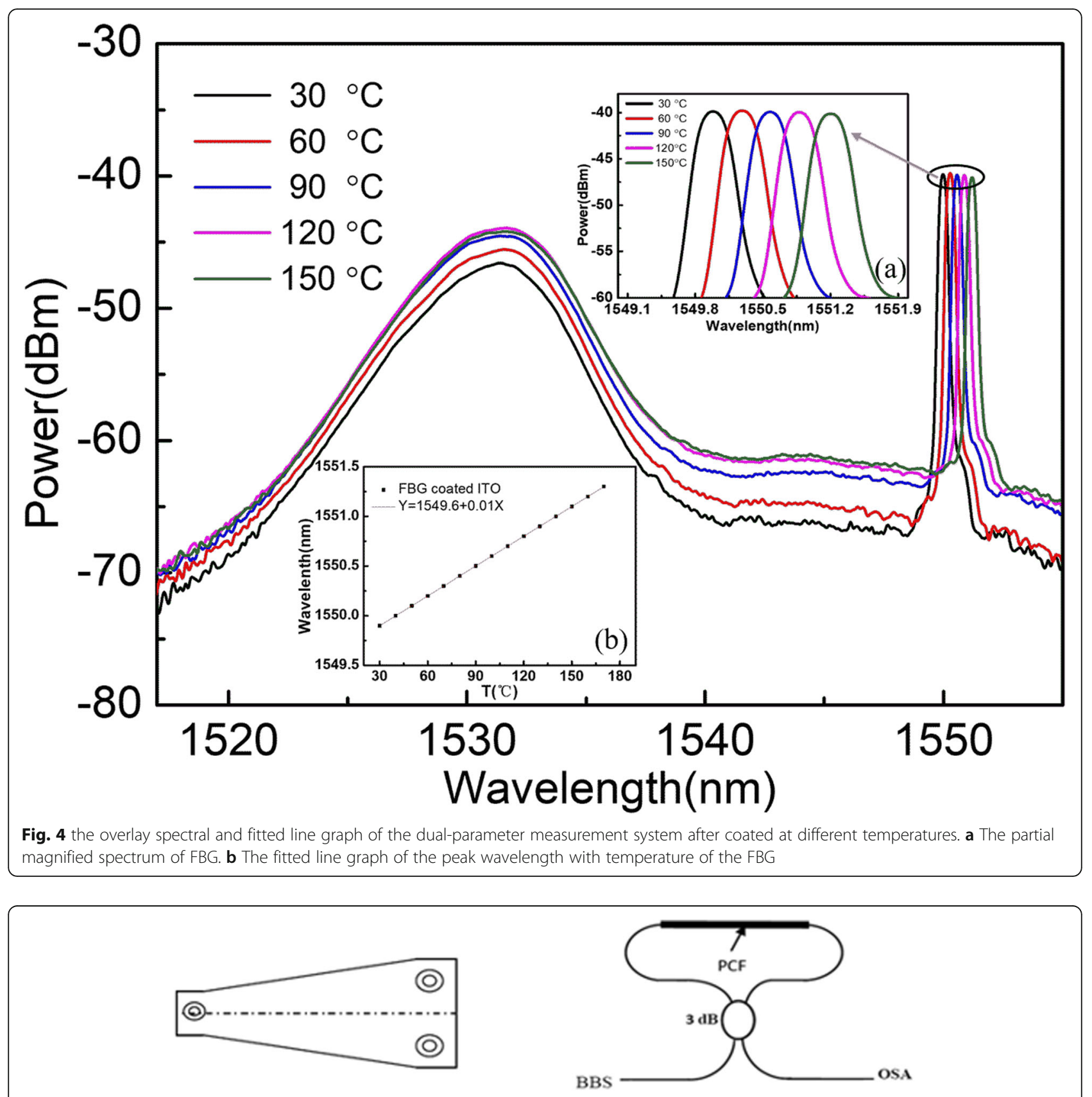

(a)

(b)

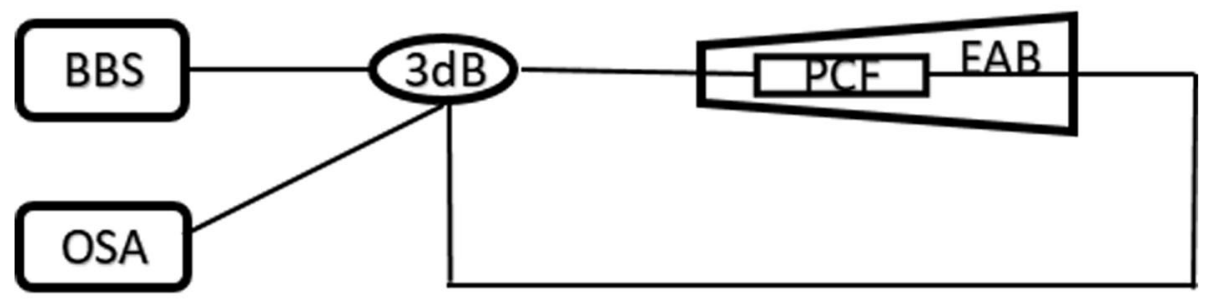

(c)

Fig. 5 Diagram of pressure sensing: a Isometric beam structure, b Photonic crystal fiber loop mirror, c The principle diagram 


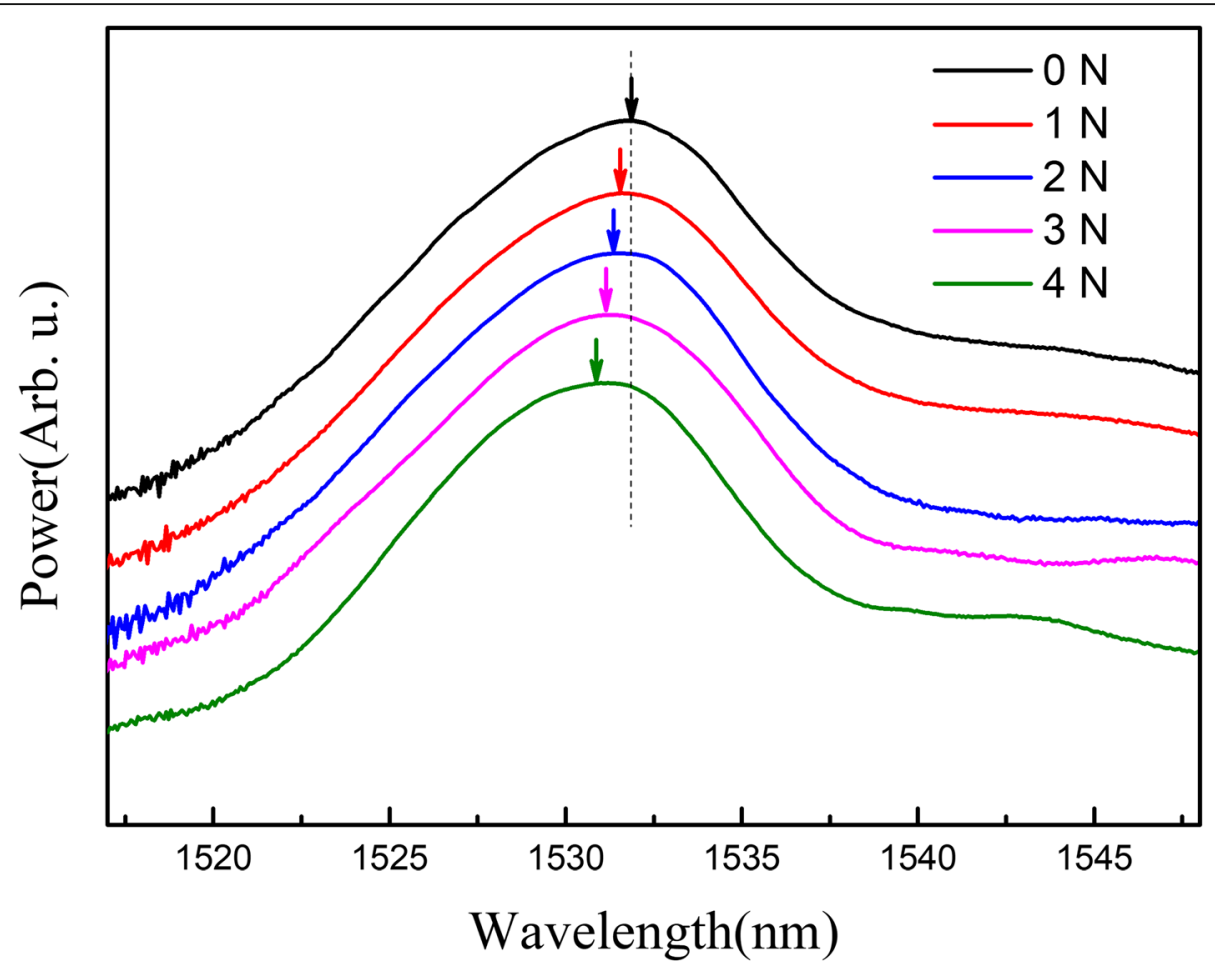

Fig. 6 The spectral curves under different pressure for connecting PCF

pressure sensing has a blue shift when the pressure is increasing but the characteristic peak of temperature does not change. The characteristic peak of FBG, which is used for temperature sensing has a red shift with the increasing temperature. Therefore, the characteristic peaks of the two sensitive parameters are shifted to opposite directions when the temperature and pressure of the dual-parameter sensor are changed at the same time, which avoids the mutual interference problem of the dual-parameter detection. Meanwhile, a metal device is designed for avoiding the influence of the stress on FBG, which is also sensitive to temperature. In this way the temperature and stress can be easily separated.
The two contrast fitting lines are the peak wavelength of PCF changing with stress increasing before connecting FBG and after, which is shown in Fig. 9. The red fitting line is before PCF connecting FBG and the black one is after connecting.

The peak wavelength sensitivity of $0.189 \mathrm{~nm} / \mathrm{N}$ and $0.150 \mathrm{~nm} / \mathrm{N}$ have been obtained based on FBG and PCF/FBG pressure sensors, which is shown in Fig. 9. The sensitivity of PCF and FBG of stress detection slightly drop due to the multiple splice losses of connecting than that of PCF, which can be improved by increasing the welding technique. As is shown in Fig. 9, there is a higher linearity of the fitted straight

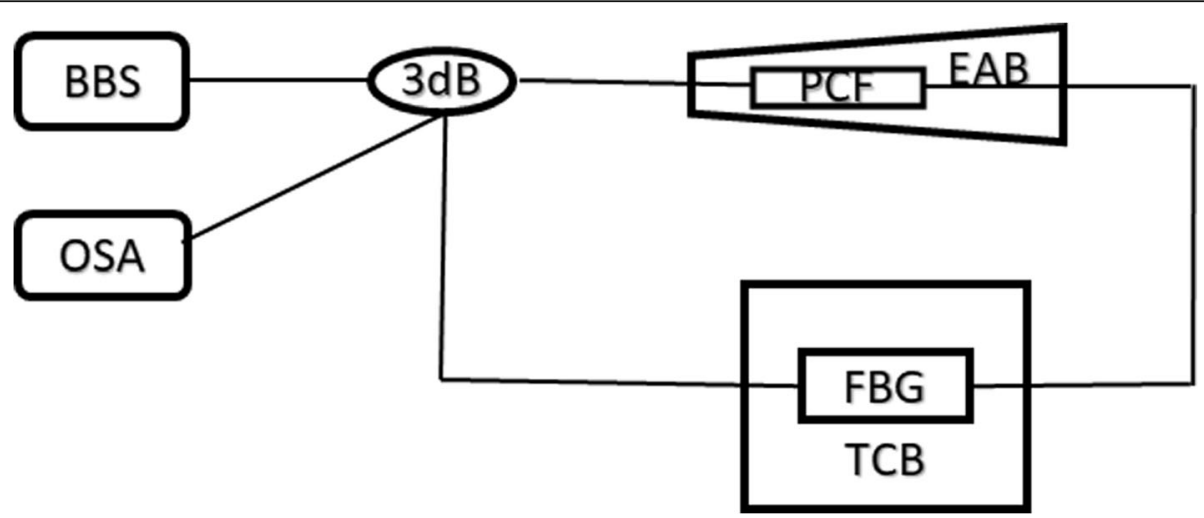

Fig. 7 The schematic diagram of optical fiber sensor of dual-parameter 


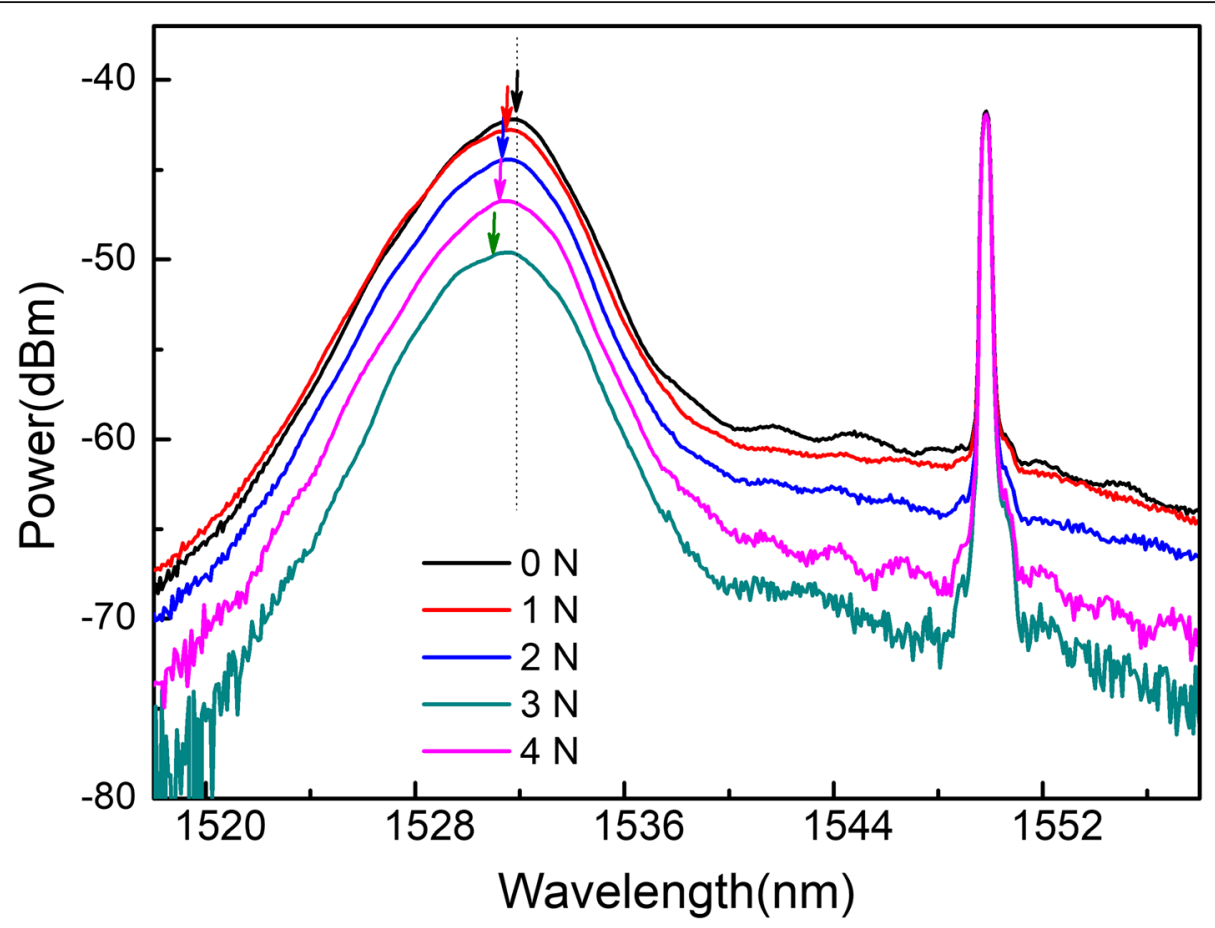

Fig. 8 The spectral curves under different pressure in connecting of PCF and FBG

line by connecting with FBG. The experimental results are consistent with the fitting curves when FBG and PCF are linked together. Therefore, dualparameter measure- ment sensor of combining between FBG and PCF have the strong reliability in application of a pressure measurement.
The wavelengths of dual-parameter measure- ments with temperature and stress are shown in Fig. 10. It is indicated that the wavelengths are changing to the opposite direction with the temperature and stress varying in the same time. The minimum distance between the temperature and stress characteristic peaks detected by

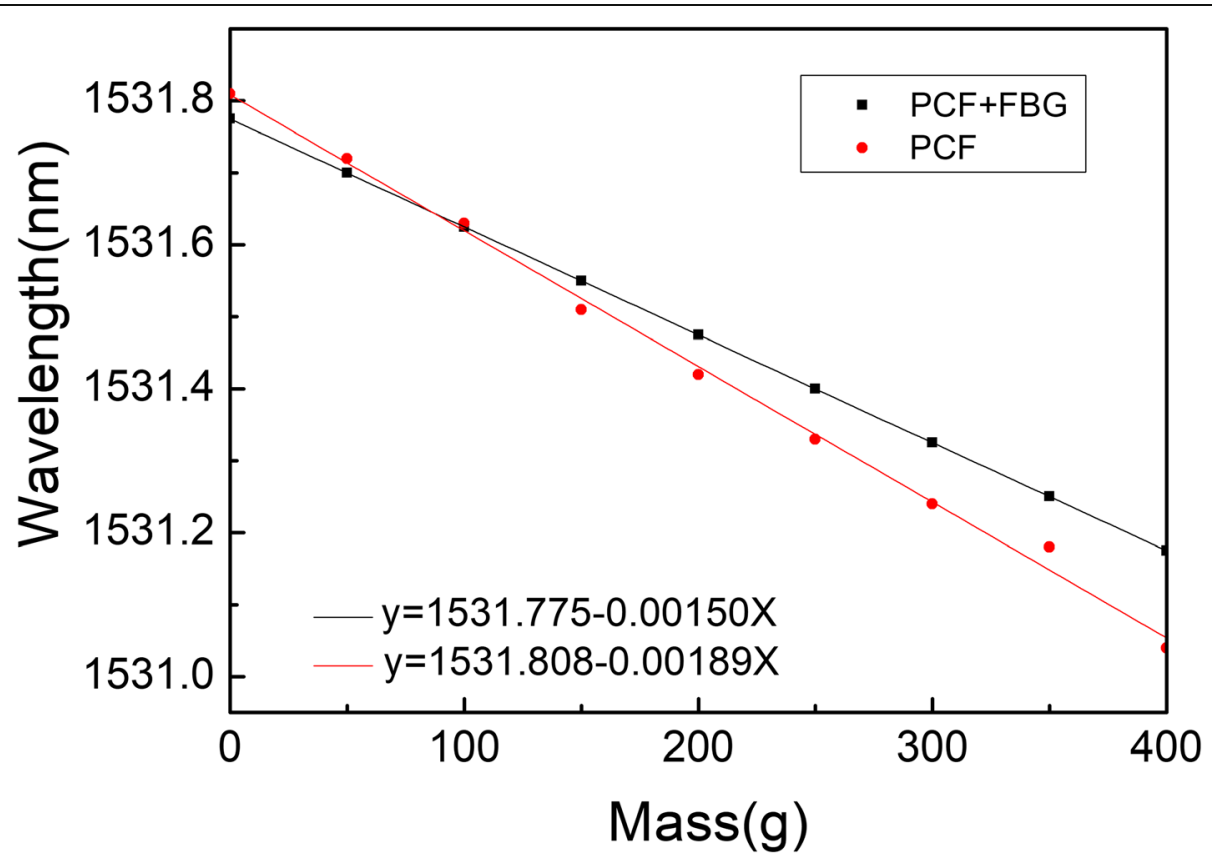

Fig. 9 Peak wavelength versus pressure mass 


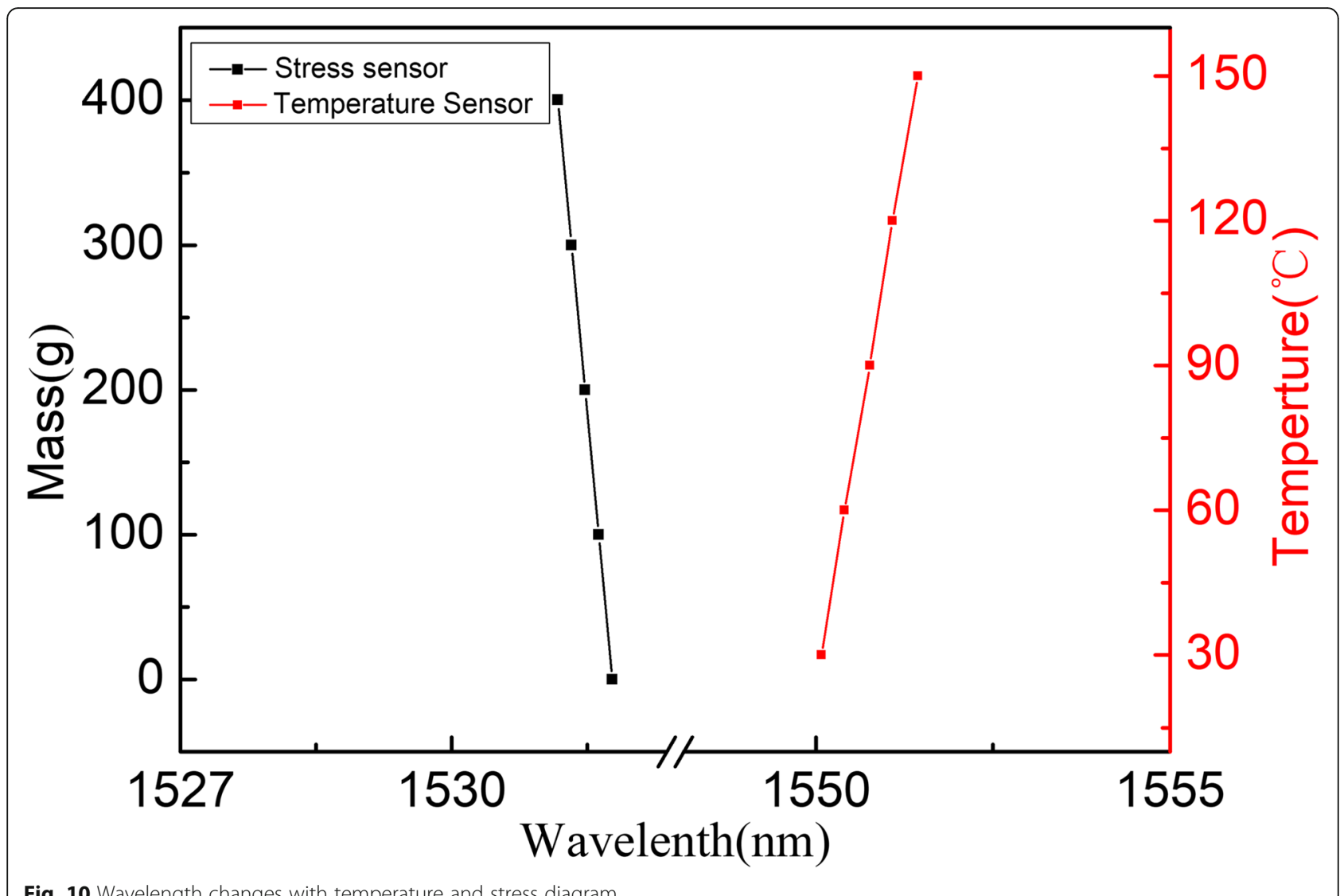

Fig. 10 Wavelength changes with temperature and stress diagram

the OSA is $18.305 \mathrm{~nm}$. Therefore, the two characteristic peaks can effectively avoid mutual interference of dualparameter. However, the Mach Zehnder interferometer needs two interference arms, which greatly limits the cascade of multi-sensor units and multi parameter sensing detection [28]. The transmission loss of FB interferometer is very large, which has a strong demand on the size of the cavity and is not conducive to long-distance transmission [13]. SPR needs precious metals, so it is costly [29]. The structure of the sensor designed by us is simple and easy to package. It can be used for two parameter and multi parameter sensors. By cascading different sensitive units, two or more specific tests, such as temperature and humidity, specific virus and cancer cell detection and protein detection, can be realized. The sensitivity is not significantly reduced and the stability is improved, so that the design idea of the system is feasible.

\section{Conclusions}

The temperature and pressure dual-parameter sensor based on Sagnac interferometer and the sensitive probes of PCF and FBG are designed in theory and experiment. The temperature sensitivity of FBG is improved from $0.0106 \mathrm{~nm} /{ }^{\circ} \mathrm{C}$ to $0.0112 \mathrm{~nm} /{ }^{\circ} \mathrm{C}$ by connecting PCF. The linearity of temperature are both 0.9986 before and after FBG connecting with PCF. The linearity of stress sensing has been in theory and experiment. The temperature sensitivity of significantly improved by connecting with FBG, and the sensitivity is not lower than $0.150 \mathrm{~nm} / \mathrm{N}$.

The characteristic peaks of the temperature and pressure are red and blue shift respectively when the dualparameter are changing at the same time, so dualparameter do not interfere to interact each other. The structure of the sensor designed by us is simple and easy to package. It can be used for two parameter or multi parameter sensors. By cascading different sensitive units, two or more specific tests, such as temperature and humidity, specific virus and cancer cell detection and protein detection, can be realized. The optical fiber sensor of multi-parameter measurement gives impetus to potential application in the optical communication, automatic control, biomedicine measurement and so on.

\section{Acknowledgments}

Tanks for supporting the National Natural Science Foundation of China under Youth Science Foundation Project (NSFC-61805005), The National Natural Science Foundation of China (NSFC-61473023).

\section{Author details}

All the authors are in College of Applied Sciences, Beijing University of Technology, No.100 Pingleyuan, Chaoyang District, Beijing 100,124, China. 


\section{Authors' contributions}

All authors contribute to the designed, discussion and preparation of the manuscript. The authors read and approved the final manuscript.

\section{Funding}

This work is funded by the National Natural Science Foundation of China under Youth Science Foundation Project (NSFC-61805005), The National Natural Science Foundation of China (NSFC-61473023).

\section{Availability of data and materials}

Please contact authors for data requests.

\section{Competing interests}

The authors declare that they have no competing interests.

Received: 4 November 2019 Accepted: 11 June 2020

Published online: 19 June 2020

\section{References}

1. $\mathrm{Xu}, \mathrm{Z}$., Shu, X., Fu, H.: Sensitivity enhanced fiber sensor based on a fiber ring microwave photonic filter with the Vernier effect. Opt. Express. 25, 2155921566 (2017)

2. Huang, Q., Yu, Y., Ou, Z., Chen, X., Wang, J., Yan, P., Du, C.: Refractive index and strain sensitivities of a long period fiber grating. Photonic Sens. 4, 92-96 (2014)

3. Zhong, X., Wang, Y., Qu, J., Liao, C., Liu, S., Tang, J., Wang, Q., Zhao, J., Yang, K., Li, Z: High-sensitivity strain sensor based on inflated long period fiber grating. Opt. Lett. 39, 5463-5466 (2014)

4. Verma, R., Gupta, B.D.: Detection of heavy metal ions in contaminated water by surface plasmon resonance based optical fibre sensor using conducting polymer and chitosan. Food Chem. 166, 568-575 (2015)

5. Minkovich, V., Sotsky, A.: Tapered photonic crystal fibers coated with ultrathin films for highly sensitive bio-chemical sensing. J. Eur. Opt. Soc. 15(1), 16 (2019)

6. Shen, C., Zhong, C., Chu, J., Zou, X., Jin, Y., Wang, J., Dong, X., Li, Y., Wang, L.: Temperature-insensitive strain sensor using a fiber loop mirror based on low-birefringence polarization-maintaining fibers. Opt. Commun. 287, 31-34 (2013)

7. Wang, S., Lu, P., Mao, L., Liu, D., Jiang, S.: Cascaded interferometers structure based on dual-pass Mach-Zehnder interferometer and Sagnac interferometer for dual-parameter sensing. Opt. Express. 23, 674-680 (2015)

8. He, H., Wang, L., Yin, L.: Asymmetric elliptical-hole dual-core photonic crystal fiber with enhanced pressure sensitivity. Opt. Fiber Technol. 20, 380-383 (2014)

9. Lee, B.: Review of the present status of optical fiber sensors. Opt. Fiber Technol. 9, 57-79 (2003)

10. Gallego, D., Lamela, H.: High-sensitivity ultrasound interferometric singlemode polymer optical fiber sensors for biomedical applications. Opt. Lett. 34, 1807-1809 (2009)

11. Homola, J.: Present and future of surface plasmon resonance biosensors. Anal. Bioanal. Chem. 377, 528-539 (2003)

12. Aray, A., Chiavaioli, F., Arjmand, M., Trono, C., Tombelli, S., Giannetti, A., Cennamo, N., Soltanolkotabi, M., Zeni, L., Baldini, F.: SPR-based plastic optical fibre biosensor for the detection of Creactive protein in serum. J. Biophotonics. 9, 1077-1084 (2016)

13. Wang, M., Yang, M., Cheng, J., Zhang, G., Liao, C., Wang, D.: Fabry-Pérot interferometer sensor fabricated by femtosecond laser for hydrogen sensing. IEEE Photonics Technol. Lett. 25, 713-716 (2013)

14. Vollmer, F., Arnold, S.: Whispering-gallery-mode bio-sensing: label-free detection down to single molecules. Nat. Methods. 5, 591-596 (2008)

15. Boleininger, A., Lake, T., Hami, S., Vallance, C.: Whispering gallery modes in standard optical fibres for fibre profiling measurements and sensing of unlabelled chemical species. Sensors. 10, 1765-1781 (2010)

16. Wang, J., Shen, C., Lu, Y., Chen, D., Zhong, C., Chu, J., Dong, X., Chan, C.: Liquid Refractive Index Sensor Based on a Polarization-Maintaining Fiber Loop Mirror. IEEE Sens. J. 13, 1721-1724 (2013)

17. Shi, J., Su, G., Xu, D., Wang, Y., Zhang, H., Fu, S., Feng, J., Yan, C., Xu, W., Yao, J.: Dual-Parameter Sensor Using a Long-Period Grating Concatenated With Polarization Maintaining Fiber in Sagnac Loop. IEEE Sens. J. 16, 4326-4330 (2016)
18. Pavlath, G.A., Shaw, H.J.: Birefringence and polarization effects in fiber gyroscopes. Appl. Optics. 21, 1752-1757 (1982)

19. Su, J.: Label-free biological and chemical sensing using whispering gallery mode optical resonators: past, present, and future. Sensors. 17, 540 (2017)

20. Yang, R., Bao, H., Zhang, S., Ni, K., Zheng, Y., Dong, X.: Simultaneous Measurement of Tilt Angle and Temperature With Pendulum-Based Fiber Bragg Grating Sensor. IEEE Sens. J. 15, 6381-6384 (2015)

21. Arasu, P., Noor, A.S.M., Shabaneh, A.A.: Absorbance properties of gold coated fiber Bragg grating sensor for aqueous ethanol. J. Eur. Opt. Soc. 9, 14018 (2014)

22. Yuan, L.L., Zhao, Y., Sato, S.Y.: Development of a low-cost and miniaturized fiber Bragg grating strain sensor system. Jpn. J. Appl. Phys. 56, 052502 (2017)

23. Dong, B., Hao, E.J: Core-offset hollow core photonic bandgap fiber-based intermodal interferometer for strain and temperature measurements. Appl. Optics. 50, 3011-3014 (2011)

24. Bock, W.J., Chen, J., Eftimov, T., Urbanczyk, W.: A photonic crystal fiber sensor for pressure measurements. IEEE T. Instrum. Meas. 55, 1119-1123 (2006)

25. Qian, W., Zhao, C., Chan, C., Hu, L., Li, T., Wong, W., Zu, P., Dong, X.: Temperature Sensing Based on Ethanol-Filled Photonic Crystal Fiber Modal Interferometer. IEEE Sens. J. 12, 2593-2597 (2012)

26. Gong, H., Chan, C.C., Zhang, Y.F., Wong, W.C., Dong, X.Y.: Miniature refractometer based on modal interference in a hollow-core photonic crystal fiber with collapsed splicing. J. Biomed. Opt. 16, 017004 (2011)

27. Hu, D.J., Lim, J.L., Jiang, M., Wang, Y., Luan, F., Shum, P.P., Wei, H., Tong, W.: Long period grating cascaded to photonic crystal fiber modal interferometer for simultaneous measurement of temperature and refractive index. Opt. Lett. 37, 2283-2285 (2012)

28. Liou, J., Yu, C.: All-fiber Mach-Zehnder interferometer based on two liquid infiltrations in a photonic crystal fiber. Opt. Express. 23, 6946-6951 (2015)

29. Mitsuhiro, I., Seki, A., Watanabe, K.: Gold thickness dependence of SPR-based hetero-core structured optical fiber sensor. Sens. Actuators B Chem. 106, 363-368 (2005)

\section{Publisher's Note}

Springer Nature remains neutral with regard to jurisdictional claims in published maps and institutional affiliations.

\section{Submit your manuscript to a SpringerOpen ${ }^{\circ}$ journal and benefit from:}

- Convenient online submission

Rigorous peer review

- Open access: articles freely available online

High visibility within the field

- Retaining the copyright to your article

Submit your next manuscript at $\boldsymbol{\nabla}$ springeropen.com 